\title{
Consenso de Reanimación Cardiopulmonar Pediátrica del Comité de RCP de la Sociedad Latinoamericana de Cuidados Intensivos Pediátricos (SLACIP). Resumen Ejecutivo
}

\author{
Latin American Consensus on Pediatric Cardiopulmonary Resuscitation. \\ An Executive Summary from the CPR Committee, Latin American Society \\ of Pediatric Intensive Care (SLACIP)
}

\begin{abstract}
Adriana Yock-Corrales ${ }^{a}$, Santiago Campos-Miño ${ }^{b}$, Raffo Escalante Kanashiroc $^{c}$, por el Comité de RCP de la Sociedad Latinoamericana de Cuidado Intensivo Pediátrico (SLACIP)
\end{abstract}

\begin{abstract}
aServicio de Emergencias. Hospital Nacional de Niños "Dr. Carlos Sáenz Herrera", CCSS. San José, Costa Rica.
bDepartamento de Pediatría, Unidad de Cuidados Intensivos Pediátricos. Hospital Metropolitano. Latin American Center for Clinical Research. Quito, Ecuador.

CDEAC - Unidad de Cuidados Intensivos. Instituto Nacional de Salud del Niño. Universidad Peruana de Ciencias Aplicadas, Universidad Nacional Federico Villarreal. Lima, Perú.
\end{abstract}

Recibido: 7 de mayo de 2021; Aceptado: 2 de septiembre de 2021

¿Qué se sabe del tema que trata este estudio?

Existen guías internacionales que se actualizan cada 5 años sobre los procedimientos de diagnóstico situacional y manejo del paro cardiorespiratorio en niños, pero su origen es de países desarrollados con datos no necesariamente cercanos a la realidad latinoamericana.
¿Qué aporta este estudio a lo ya conocido?

El Consenso Latinoamericano de RCP Pediátrica revisa las guías internacionales e introduce elementos útiles propios a la realidad latinoamericana, especialmente en las áreas de ética, docencia, investigación futura, manejo prehospitalario, tratamiento del síndrome postparo, pronóstico neurológico y reanimación neonatal en tiempos de pandemia.

\section{Resumen}

La Reanimación Cardiopulmonar (RCP) es un proceso crítico con una potencial repercusión sobre la vitalidad y la funcionalidad futura del paciente. En este documento revisamos diferentes aspectos sobre diagnóstico, manejo y pronóstico de la RCP pediátrica desde un punto de vista pragmático y una visión regional desarrollada por el grupo de expertos que conforman el Comité de RCP de la Sociedad Latinoamericana de Cuidado Intensivo Pediátrico (SLACIP). Se han contemplado temas considerados principales, en especial para la mejoría del manejo del síndrome post-reanimación
Palabras clave:

Resucitación

Cardiopulmonar;

Paro Cardiaco;

Síndrome Postparo;

Pediatría

Correspondencia:

Santiago Campos Miño

drsantiagocampos@gmail.com 
y neuropronóstico postparo y para la identificación de oportunidades de investigación. La fuente principal de este Resumen Ejecutivo es el documento publicado el 2020 por el International Liaison Committee on Resuscitation, ILCOR. Ello nos ha ayudado a entender la morbimortalidad asociada y el impacto con respecto al manejo en los planes de recuperación y rehabilitación del paciente pediátrico después de la RCP. El Consenso de RCP de la SLACIP ofrece una revisión narrativa sobre aspectos en reanimación cardiopulmonar pediátrica con prioridad en el manejo crítico de estos pacientes.

\section{Abstract}

Cardiopulmonary Resuscitation (CPR) is a critical procedure with potential consequences over future vitality and functionality in survivors. In this document, we present a pragmatic and regional point of view on diagnosis, management, and prognostication in pediatric CPR developed by the Committee on CPR of the Latin American Society of Pediatric Intensive Care (SLACIP). We have chosen the main CPR topics with the aim of contribute for a better prevention and management of CPR, standardize the management of the post-cardiac arrest syndrome and neuro-prognostication, and identify opportunities for regional research. We have followed the Executive Summary published in 2020 by the International Liaison Committee on Resuscitation, ILCOR, adapted it to our reality, and developed this narrative review of pediatric CPR with the contribution of Latin American experts.
Keywords:

Cardiopulmonary

Resuscitation;

Cardiac Arrest;

Post-Cardiac Arrest

Syndrome;

Pediatrics

\section{Evidencia y Fuentes}

El Consenso de RCP de la SLACIP se ha desarrollado utilizando un modelo de revisión narrativa sobre RCP Pediátrica. La búsqueda, obtención y selección de literatura se realizó utilizando diferentes recursos electrónicos de evidencia científica e información médica. El proceso de revisión y selección de artículos no obligó a los autores a restricción en el idioma; tampoco se limitó la búsqueda por fecha de publicación. Los artículos y estudios que se seleccionaron son fundamentalmente orientados al segmento pediátrico. Un documento de referencia principal es la Guía del International Liaison Committee on Resuscitation (ILCOR) realizada el 2020. Como toda revisión narrativa, la elección de los artículos se dejó a criterio y selección de los autores, evitando en lo posible sesgo y conflicto de interés ${ }^{1}$. El presente documento denominado Consenso RCP de la SLACIP, Resumen Ejecutivo, propone recomendaciones y sugerencias en cada capítulo tratado.

\section{Secciones y Recomendaciones}

\section{Principios éticos en RCP}

La RCP es una situación clínica muy compleja con numerosos e importantes problemas éticos que cambian en el tiempo y de acuerdo con condicionantes culturales, sociales y religiosas ${ }^{2}$. Los problemas éticos en el niño tienen características muy especiales porque las decisiones, en la mayor parte de los casos, no pue- den ser tomadas por el propio paciente. Por otra parte, hay que tener en cuenta que muchas de las decisiones críticas del personal de salud se fundamentan en el conocimiento, la experiencia, el sentido común, la intuición, y en el principio médico "primum non nocere"; estos factores tienen connotaciones a nivel de legislación global y regional, y consideraciones vinculantes no legales.

\section{Recomendaciones}

- Cuándo empezar o cuándo no empezar la RCP: recomendamos no utilizar ningún criterio absoluto único para decidir el inicio o no inicio de la RCP. El equipo de reanimación debe valorar las circunstancias particulares de cada caso. Recomendamos iniciar la RCP cuando existan dudas sobre la duración del paro cardiaco (PC), la existencia de signos de muy mal pronóstico, la orden de no-reanimación, o la presencia de signos de muerte evidente, teniendo en cuenta, si es posible, los deseos de los padres ${ }^{3}$.

- Orden de no-reanimación (ONR): recomendamos que, en la página inicial del expediente clínico del paciente, cuando las circunstancias así lo justifiquen, conste claramente identificada la ONR correctamente llenada y firmada. La ONR debe ser consensuada entre el equipo de salud y los padres o responsables legales del niño, y puede ser revocada por cualquiera de las partes en cualquier momento. El niño menor de edad maduro, según sus circunstancias, debe ser informado y mostrar su aceptación con la ONR. La existencia de una ONR 
no implica la disminución del resto de los cuidados del niño, pero debe llevar consigo el establecimiento de un programa de cuidados paliativos.

- Duración de la RCP y criterios de finalización de la RCP: no podemos recomendar un único criterio para indicar la finalización de la RCP en niños. En cada caso el responsable médico de la RCP debe tener en cuenta diversos factores asociados al pronóstico, como la enfermedad y estado clínico previos, la duración del PC antes del inicio de la RCP, el ritmo electrocardiográfico al inicio de la RCP, la posibilidad de iniciar oxigenación por membrana extracorpórea (ECMO) durante la RCP, los deseos de los padres y, sobre todo, la duración de la RCP.

- ECMO y RCP: recomendamos que, en los centros hospitalarios en los que exista la posibilidad de realizar ECMO durante la RCP, se establezcan protocolos, se prepare el material y se realice el entrenamiento del personal para instaurar esta técnica de forma rápida y precoz, fundamentalmente en los niños con PC de etiología cardiaca que no respondan en los primeros minutos a las maniobras de RCP. No recomendamos instaurar ECMO durante la RCP en centros hospitalarios sin programa específico de ECMO en RCP. Recordar que en pediatría no tenemos evidencia suficiente para una recomendación formal.

- Transporte pediátrico y RCP: recomendamos realizar el transporte de un niño mientras se realiza la RCP si se puede garantizar una RCP adecuada durante el transporte y el niño se puede beneficiar de un tratamiento vital en otra institución (por ejemplo ECMO, cirugía, canalización vascular $)^{4}$.

- Presencia de padres y familiares durante la RCP: recomendamos que en cada situación se valore la posibilidad, beneficios e inconvenientes de la presencia de los padres o familiares durante la RCP. Es necesario valorar los deseos de los padres y del personal sanitario presente durante la RCP, así como la repercusión de la presencia de los padres sobre la actuación del personal y la calidad de la RCP.

- Donación de órganos tras la RCP y donación en asistolia: recomendamos que todos los pacientes que han recuperado la circulación espontánea tras un PC y posteriormente queden en muerte cerebral, sean valorados como donantes potenciales de órganos. Recomendamos que cada centro hospitalario valore la posibilidad de establecer programas de donación en asistolia para los niños que se encuentran en una situación clínica irreversible sin estar en muerte cerebral. Cada país debe establecer las regulaciones adecuadas para realizar programas de donación en asistolia controlada ${ }^{5}$.

- Docencia y RCP: recomendamos realizar la formación y el entrenamiento en RCP pediátrica a todo el personal sanitario que trabaja con niños y a la población general con métodos de simulación clínica. Recomendamos utilizar la grabación de la RCP para mejorar la calidad y la docencia del procedimiento, solicitando a los Comités de Ética la exención del consentimiento informado, especificando la utilización de la grabación exclusivamente para fines asistenciales y docentes. Recomendamos que el entrenamiento de maniobras de RCP en pacientes recién fallecidos solamente se realice en programas docentes bien establecidos y bajo la supervisión de un especialista. La exención del consentimiento para esta práctica debe ser previamente establecida por un Comité de Ética y este concepto debe ser claramente definido.

- Investigación y RCP: recomendamos no extrapolar directamente los resultados de estudios de investigación de RCP en adultos a la RCP pediátrica, sin realizar previamente los estudios clínicos en niños que comprueben sus resultados. Recomendamos realizar, siempre que sea posible, estudios de investigación en modelos de simulación o modelos animales pediátricos, previamente a los estudios clínicos de RCP en niños. Recomendamos la concesión de la exención del consentimiento informado o el consentimiento diferido para los estudios clínicos de PC y RCP en niños.

\section{Eslabones de la Cadena de Sobrevida}

Los eslabones de la cadena de sobrevida nos recuerdan que la prevención de muertes en niños por lesiones o enfermedad es una responsabilidad compartida entre la comunidad, cuidadores y personal de salud. Es necesario educar a la población para promover la prevención de accidentes y la detección oportuna de signos de alerta, para acudir en forma temprana a los servicios de Emergencia. El uso del desfibrilador externo automático (DEA) tiene cabida en ambos escenarios de $\mathrm{PC}$, pero probablemente sea de mayor utilidad en el PC intrahospitalario (PCIH). Se deben establecer sistemas de evaluación rutinaria en salas de hospitalización para detectar tempranamente las condiciones de riesgo, así como conformar equipos de respuesta rápida para una intervención eficiente con potencial de prevenir el PC. Desde la actualización 2015, la cadena de supervivencia pediátrica incluye los siguientes eslabones: a) prevención del PC; b) RCP temprana de alta calidad realizada por un testigo; c) activación rápida de sistemas de emergencia y solicitud del DEA mientras se continúa con la RCP; d) soporte vital avanzado (SVA) que incluye la estabilización y traslado a una unidad de cuidados posteriores al PC o unidad de cuidados intensivos (UCI); y, e) manejo y cuidados posteriores al $\mathrm{PC}^{6}$. En las últimas guías de reanimación de American Heart Association se mencionan los eslabones de sobre- 
vida pediátrica para el PCIH y para el PC extrahospitalario $(\mathrm{PCEH})$. Ambos hacen énfasis en la importancia de la prevención del PC y se agrega un sexto eslabón que es el de "Recuperación"7.

\section{Recomendación}

- Se recomienda que la conceptualización de los Eslabones de Cadena de Sobrevida sea una filosofía de conducta y acción ante un evento de PCR, sea intrahospitalario o extrahospitalario.

\section{Uso del DEA}

El DEA es un dispositivo que, mediante la colocación de parches en el pecho del paciente, es capaz de analizar el ritmo cardiaco y administrar una descarga eléctrica cuando detecta un ritmo desfibrilable. De la misma manera, el DEA indica en forma audible y/o escrita la secuencia correcta del procedimiento de desfibrilación y RCP; y, en algunos dispositivos, puede brindar retroalimentación durante la reanimación.

\section{Recomendaciones}

- Niños de 1 a 8 años: recomendamos que el DEA posea un atenuador de descarga y parches pediátricos. Algunos DEA tienen un interruptor para administrar una descarga pediátrica, pero debe activarse al momento de su utilización. En otros modelos el atenuador se incorpora a los parches pediátricos ${ }^{8}$.

- Lactantes: recomendamos el uso de un desfibrilador manual. Si no se dispone de éste, se puede utilizar un DEA con atenuador y, de no tenerlo disponible, se puede utilizar un DEA sin atenuador.

- Sobre el uso de parches: si no se cuentan con parches pediátricos se pueden utilizar los parches de adulto, pero éstos no deben tocarse entre sí. Nunca se deben cortar los parches de adulto para colocarlos en un niño. En el niño pequeño se puede colocar un parche en la región frontal del tórax y el otro en la espalda.

\section{Soporte Vital Avanzado Pediátrico}

Cada país debe diseñar su propio algoritmo basado en las recomendaciones internacionales para asegurar una RCP de alta calidad en todo momento. Se debe poner énfasis en la prevención y atención precoz de las patologías que llevan al deterioro respiratorio y/o hemodinámico de la población pediátrica ${ }^{8,9}$.

\section{Recomendaciones}

- Vía aérea y ventilación: recomendamos proporcionar una ventilación a presión positiva (VPP) que eleve suavemente el pecho y evite la hiperinsuflación y la hiperventilación, manteniendo una frecuencia de 20 a 30 ventilaciones por minuto, adecuando esta al contexto clínico del paciente. En pacientes no intubados, se debe coordinar la VPP con las compresiones y, en los pacientes con vía aérea avanzada, se realizan compresiones continuas sin hacer pausas para las ventilaciones. Los dispositivos utilizados pueden ser bolsa y máscara, o bolsa y tubo traqueal, por lo que es recomendable el entrenamiento en las técnicas para instrumentar la vía aérea según la disponibilidad local de material. Se pueden utilizar tubos traqueales sin o con balón; sin embargo, recomendamos los tubos con balón para reducir la fuga de aire y reducir la necesidad del recambio posterior. La evidencia disponible no respalda el uso rutinario de atropina antes de la intubación en lactantes y niños críticamente enfermos, pero puede ser razonable su utilización como premedicación en intubaciones de emergencia cuando existe un mayor riesgo de bradicardia ${ }^{10}$. Recomendamos, en lo posible, que la monitorización respiratoria incluya oximetría de pulso, $\mathrm{CO}_{2}$ espirado, gases en sangre y radiografía de tórax.

- Circulación: recomendamos que la monitorización circulatoria incluya ECG, presión arterial invasiva, $\mathrm{CO}_{2}$ espirado, ecografía y, de ser posible, espectrometría cercana al infrarrojo (NIRS). Sin embargo, la imposibilidad de contar con estos recursos no limita la capacidad de administrar RCP de alta calidad. Es razonable la administración de adrenalina durante el PC para ayudar a restaurar la circulación espontánea al aumentar la presión de perfusión coronaria y mantener la perfusión cerebral. Recomendamos su administración durante la RCP de ritmos no desfibrilables en los primeros cinco minutos de RCP9 . En todo niño que presente una arritmia recomendamos la comprobación de signos vitales y pulso central. Si no hay signos vitales se debe iniciar RCP; si tiene signos vitales y pulso central debe evaluarse el estado hemodinámico. En la fibrilación ventricular (FV) o taquicardia ventricular sin pulso (TVSP) luego de dos desfibrilaciones, se recomienda la utilización de la estrategia RCP-Droga-Desfibrilación, deberá iniciarse con adrenalina y en caso de persistencia, recomendamos el uso indistinto amiodarona o lidocaína. La administración de drogas durante la RCP enfatiza la necesidad de la colocación de un acceso vascular, por ello se requiere material idóneo y entrenamiento para la colocación de un acceso venoso o intraóseo.

\section{Reanimación neonatal}

El minuto de oro es el tiempo inmediatamente después del nacimiento durante el cual deberemos evaluar al recién nacido (RN) e iniciar la VPP cuando esté indicada. Si bien el Apgar es un indicador de vitalidad y muchos de sus componentes se utilizan en la evalua- 
ción inicial del RN, no es la escala que nos indica si debemos o no realizar maniobras de reanimación, sino más bien nos permite verificar el estado fisiológico del neonato y su respuesta a la reanimación ${ }^{11,12}$.

\section{Recomendaciones}

- Pasos iniciales: cuando nace el bebé, recomendamos valorar el tono muscular, si respira o llora, y si es de término; si estas tres respuestas son positivas, los pasos iniciales pueden realizarse sobre el regazo de la madre. Si alguna de estas respuestas es negativa, recomendamos el uso de una cuna de calor radiante para evaluar la frecuencia cardíaca y el esfuerzo respiratorio mientras se realizan los pasos iniciales.

- Vía aérea y ventilación: recomendamos iniciar la VPP durante el minuto de oro en los niños que no han podido establecer una respiración regular normal o que su frecuencia cardiaca (FC) sea menor a 100/min. La VPP es la principal intervención en la reanimación neonatal ${ }^{11}$. La reanimación del RN de término o prematuro con oxígeno al $21 \%$, en comparación con $100 \%$, tiene una menor mortalidad sin que existan diferencias en el desarrollo neurológico de los sobrevivientes.

- Circulación: si la FC es menor a $60 /$ min a pesar de VPP efectiva durante 30 segundos, idealmente con una vía aérea segura, recomendamos iniciar compresiones torácicas $^{12}$. Si a pesar de un minuto de compresiones torácicas coordinadas con ventilación efectiva y oxígeno al 100\% la FC se mantiene debajo de 60/min, recomendamos administrar adrenalina idealmente a través de un acceso vascular o intraóseo. En la bradicardia persistente a pesar de ventilación, compresiones y adrenalina debe descartarse hipovolemia o neumotórax.

- Entrenamiento: recomendamos una preparación continua de los profesionales sanitarios en reanimación neonatal, dado que la aplicación de prácticas estandarizadas ha mejorado el pronóstico neonatal. En los países en desarrollo, la reanimación neonatal estandarizada, comparada con la atención neonatal básica, ha reducido la mortalidad neonatal temprana y a los 28 días. Recomendamos evaluar métodos educacionales innovadores que mejoren el conocimiento, las habilidades y el comportamiento de trabajo en equipo ${ }^{13}$.

\section{RCP en circunstancias especiales}

Las situaciones especiales son eventos infrecuentes en los que el algoritmo estándar de RCP debe ser modificado. Dentro de las situaciones especiales se consideran el trauma, situaciones peri-quirúrgicas, unidades de hemodiálisis, aviones comerciales, embarazo, niños con necesidades especiales, entre otras.

\section{Recomendaciones}

- Recomendamos que cada región o centro hospitalario estandarice su respuesta frente a las circunstancias especiales que más probabilidad tienen de ocurrir con base a la zona geográfica, tipo de centro de salud, nivel de complejidad o cualquier otra peculiaridad. Es aconsejable el uso de ayudas cognitivas para estas situaciones.

- La educación a través de cursos estandarizados, simulación, entrenamiento rápido y frecuente, debriefing post-evento, implementados aisladamente o en combinación, puede mejorar la calidad de la RCP brindada a los pacientes con circunstancias especiales $^{8,14}$.

\section{Aspectos de RCP prehospitalaria}

La incidencia de PCEH pediátrico es baja (8 por 100.000 personas/año), con mayor incidencia en los lactantes menores de 1 año en comparación con otros grupos de edad (lactantes 75,3, niños 3,7 y adolescentes $6,3$ por 100.000 personas/año, $\mathrm{p}<0,001)^{15}$. La mayoría de los PCEH pediátricos ocurren en el hogar (69\%), el $68 \%$ de los testigos son miembros de la familia e iniciaron la reanimación un 33\%.

\section{Recomendaciones}

- Recomendamos enfatizar las estrategias de prevención para disminuir los eventos relacionados con el PCEH en la población pediátrica; dado que la mayoría de los eventos ocurren en el hogar, se requiere la educación de las familias como primeros intervinientes en la RCP después de PCHE.

- Dado que el PC pediátrico es mayoritariamente de origen respiratorio, recomendamos priorizar la RCP con compresiones y ventilaciones; sin embargo, el DEA debe usarse cuando esté disponible.

- Sugerimos el uso de ventilación con bolsa-máscara en lugar de la intubación traqueal o la inserción de un dispositivo supraglótico para el manejo de la vía aérea en niños con PCEH.

- Recomendamos garantizar RCP de calidad en los servicios de atención prehospitalaria. Esto puede lograrse con el uso de dispositivos de retroalimentación de la calidad de las compresiones, a través de estrategias educativas como el entrenamiento corto y frecuente, simulación, debriefing post-evento, o la combinación de varias de estas estrategias.

- Recomendamos favorecer la investigación de la RCP después de PCEH y los resultados al alta en Latinoamérica para hacer en el futuro recomendaciones adaptadas al contexto local.

\section{Síndrome Post-Paro}

Definen el síndrome post-paro (SPP) los siguientes fenómenos ${ }^{16,17}$ : lesión cerebral anóxica, disfunción 
miocárdica relacionada al paro, respuesta sistémica de isquemia/reperfusión, y patología precipitante persistente. Se describen 4 fases en el SPP y en ellas tenemos prioridades de tratamiento con un enfoque multisistémico en cada fase ${ }^{18}$. El manejo del SPP incluye un paquete de medidas (bundle, por su nombre en inglés) que ha demostrado mejores resultados en términos de sobrevida y desenlace neurológico. Las recomendaciones que se presentan provienen, en su mayor parte, de las actualizaciones 2019 de American Heart Association (CPR \& ECC Guidelines) ${ }^{8,18}$.

\section{Recomendaciones}

- Ventilación: recomendamos mantener normooxigenación y normo-ventilación. Los objetivos recomendados son $\mathrm{SpO}_{2}$ entre 94 y 98\%, y $\mathrm{PaCO}_{2}$ de $35-45 \mathrm{mmHg}$, con la ayuda de capnografía continua. Mientras el paciente se encuentre en ventilación mecánica $(\mathrm{VM})$, recomendamos usar una estrategia protectora: $\mathrm{FIO}_{2}<0,6$, presión de meseta $<30 \mathrm{cmH}_{2} \mathrm{O}$ y presión sobre PEEP menor a 16 $\mathrm{cmH}_{2} \mathrm{O}$.

- Circulación: recomendamos implementar una monitorización hemodinámica invasiva con los objetivos de alcanzar euvolemia, normotensión, gasto cardiaco adecuado y transporte de oxígeno óptimo a los tejidos. Debe vigilarse la perfusión distal, la diuresis, la presencia de arritmias, la corrección de la acidosis y el aclaramiento de lactato. La hemoglobina podría mantenerse cercana a $10 \mathrm{~g} /$ dL para optimizar el contenido arterial de oxígeno.

- Manejo neurológico: recomendamos el control de convulsiones idealmente con la implementación de EEG continuo. La analgesia-sedación debe considerarse en niños en VM mecánica o para el control de los escalofríos. Para no interferir con el examen neurológico, debe seleccionarse medicación con efecto de corta duración. Recomendamos realizar un EEG en forma precoz y, de ser posible, mantener una monitorización EEG continua. Los fluidos intravenosos deben ser isotónicos y el objetivo de natremia es alrededor de $145 \mathrm{mEq} / \mathrm{L}$. La glicemia puede mantenerse en rangos de $80-180$ $\mathrm{mg} / \mathrm{dL}$.

- Control térmico: recomendamos un control estricto de la temperatura (normotermia activa) o el uso de hipotermia controlada mientras se mantiene la homeostasis metabólica.

- Pronóstico: la evaluación del pronóstico requiere un seguimiento clínico frecuente tomando en consideración el tiempo desde el PC, uso de medicación, y uso de hipotermia controlada (tabla 1). Recomendamos que la evaluación del pronóstico se realice mediante la participación de un equipo multidisciplinario. La evaluación del pronóstico, diagnóstico de muerte encefálica y decisiones para pasar a cuidados de final de la vida deben tener en cuenta la legislación propia de cada país.

\section{Control de calidad de la RCP}

El retorno a la circulación espontánea, la supervivencia y una evolución neurológica favorable dependen de la calidad de la RCP. Esta es la razón por la que los científicos de la RCP siempre buscan más estrategias para incrementar la sobrevida después del PC y unen esfuerzos para optimizar su calidad ${ }^{16}$. Después de un PC la sobrevida depende de un reconocimiento temprano, de la activación del sistema de respuesta a emergencia y de la calidad de RCP brindada ${ }^{15}$.

\section{Recomendaciones}

La RCP de calidad se caracteriza por

- Comprimir rápido: compresiones con frecuencia de 100 a 120/min.

- Comprimir fuerte: profundidad de $4 \mathrm{~cm}$ en lactantes y $5 \mathrm{~cm}$ en niños.

- Minimizar las interrupciones.

- Permitir la expansión completa del tórax entre las compresiones (evitar la compresión residual del tórax).

- Evitar la hiperventilación.

La calidad de la RCP se puede monitorizar mediante indicadores fisiológicos, que informan de la evolución del paciente, e indicadores de rendimiento de la $\mathrm{RCP}$, que corresponde a la actuación de los reanimadores.

\section{Registro en RCP Pediátrica}

ILCOR, a principios de los 2000, se realizó una revisión de las experiencias y prácticas aplicadas por el uso del estilo Utstein. Esto permitió que se simplificara y se actualizara la versión de modo que fuese aplicable tanto para la resucitación de adultos como pediátrica o neonatal, así como en el medio extrahospitalario e intrahospitalario ${ }^{19}$. El estilo Utstein proporciona pautas uniformes para informar sobre la investigación de soporte vital avanzado $y$, por lo tanto, puede mejorar la atención y los resultados del paciente.

\section{Recomendamos registrar las siguientes variables según el estilo Utstein ${ }^{20,21}$}

- Relacionadas con el hospital: nivel, número de ingresos totales, número de camas hospitalarias y las dedicadas a cuidados intensivos y urgencias.

- Relacionadas con las características de los pacientes: edad, sexo, antecedentes de RCP, ingreso hospitalario o en la UCI, lugar del PC y testigo del PC.

- Relacionadas con el estado previo al evento: tipo de paciente y tipo de enfermedad. 
Tabla 1. Factores pronósticos que se pueden observar antes o durante el paro cardiaco en niños

\begin{tabular}{|c|c|c|c|}
\hline Fase & Factor & Desenlace & Sobrevida \\
\hline \multirow{8}{*}{$\begin{array}{l}\text { Antes } \\
\text { del paro } \\
\text { cardiaco }\end{array}$} & Antecedente & & \\
\hline & $\begin{array}{l}\text { Condición de base (sepsis, genética, metabólica, insuficiencia renal o hepá- } \\
\text { tica, cardiopatía congénita, enfermedad neurológica de base, enfermedad } \\
\text { hematológica, oncológica o inmunológica }\end{array}$ & Sobrevida al alta & Disminuida \\
\hline & Enfermedad de base de pulmón o vía aérea & Sobrevida al alta & Aumentada \\
\hline & Postoperatorio general o de corazón & Sobrevida al alta & Aumentada \\
\hline & Intervención: intubación traqueal, uso de vasopresores & Sobrevida al alta & Disminuida \\
\hline & Causa del paro & & \\
\hline & Síndrome de muerte súbita, trauma & Sobrevida a 1 año & Disminuida \\
\hline & Asfixia por inmersión, asma & Sobrevida al alta & Aumentada \\
\hline \multirow{14}{*}{$\begin{array}{l}\text { Durante } \\
\text { el paro } \\
\text { cardiaco }\end{array}$} & Paro presenciado & Sobrevida al alta & Aumentada \\
\hline & Ritmo de paro & & \\
\hline & FV o TV sin pulso (PCEH) & Sobrevida al alta o a 1 mes & Aumentada \\
\hline & AESP, bradicardia $(\mathrm{PCIH})$ & Sobrevida al alta & Aumentada \\
\hline & AESP, asistolia (PCEH) & Sobrevida al alta & Disminuida \\
\hline & Tiempo más corto hasta la descarga (PCEH) & $\begin{array}{l}\text { Sobrevida al mes con mejor } \\
\text { estado neurológico }\end{array}$ & Aumentada \\
\hline & Características de la RCP & & \\
\hline & Menos dosis de adrenalina & Sobrevida al alta & Aumentada \\
\hline & Menos tiempo para la primera dosis de adrenalina & Sobrevida al alta & Aumentada \\
\hline & $\begin{array}{l}\text { Presión diastólica > } 25 \mathrm{mmHg} \text { en lactantes y > } 30 \mathrm{mmHg} \text { en niños durante } \\
\text { la RCP }\end{array}$ & Sobrevida al alta & Aumentada \\
\hline & Profundidad adecuada de las compresiones & Sobrevida al alta & Aumentada \\
\hline & Uso de drogas durante la RCP: adrenalina, bicarbonato, calcio, atropina & Sobrevida al alta & Disminuida \\
\hline & Intubación durante la RCP & Sobrevida al alta & Disminuida \\
\hline & Mayor duración de la RCP & Sobrevida al alta & Disminuida \\
\hline
\end{tabular}

FV=fibrilación ventricular; TV = taquicardia ventricular; $\mathrm{PCIH}=$ paro cardiaco intrahospitalario; $\mathrm{PCEH}=$ paro cardiaco extrahospitalario; AESP = actividad eléctrica sin pulso; RCP=resucitación cardiopulmonar. Adaptado de: Topjian AA, de Caen A, Wainwright MS, et al. Pediatric Post-Cardiac Arrest Care A Scientific Statement From the American Heart Association. Circulation 2019; 140: e194-e233.

- Relacionadas con el episodio de PC: fecha, causa, constantes al iniciar la RCP, atención inicial recibida (RCP básica y avanzada), ritmo eléctrico, tiempos e intervalos de actuación (del PC, de inicio de $\mathrm{RCP}$, de los diferentes procedimientos, de la recuperación del pulso y del fin de la RCP), y resultados iniciales tras la RCP.

- Relacionada con el proceso post-reanimación: manejo especifico de la temperatura, evitar hipertermia, coronariografía o terapias de reperfusión.

- Relacionadas con los resultados: porcentaje de sobrevida, calidad de vida post-PC.

En Latinoamérica, las publicaciones son escasas respecto a registros en PC. En la población pediátrica no existe ningún registro activo, aunque sí hay algunos es- tudios de PC que han implementado el registro según el estilo Utstein durante varios años ${ }^{20}$. Recomendamos que las organizaciones que tratan pacientes con PC recopilen resultados y datos de procesos de atención médica. El sistema para la recolección de datos debe ser sencillo e incluir por lo menos la información básica e idealmente los complementarios, como podría ser el formato de información abreviada estilo Utstein ${ }^{19}$. Recomendamos que los hospitales que manejan pacientes pediátricos deben elaborar un registro de RCP basado en el estilo Utstein agregando la letra " $P$ " cuando se trate exclusivamente de registro de PC pediátrico. Los formatos de recolección deben basarse en términos homologados en los Descriptores en Ciencias de la Salud (DeCS). El registro debe incluir los datos tras la recuperación de la circulación espontánea y la evolución a largo plazo. 


\section{Educación, Programa, Entrenamiento y Simulación}

El entrenamiento en RCP es fundamental para mejorar la tasa de supervivencia de un PC. A pesar de los avances en la formación en RCP, las tasas de supervivencia del PC siguen siendo subóptimas tanto en los entornos hospitalarios como extrahospitalarios ${ }^{22,23}$.

\section{Recomendaciones}

- Promover que todo el personal de salud tenga un curso de reanimación ofrecido por alguna institución acreditada que garantice la entrega de conocimientos y habilidades mínimas para ser garantía de un adecuado estándar.

- Incentivar el aumento de cobertura de entrenamiento en RCP a nivel de la comunidad para aumentar la posibilidad de reanimación por testigos de un PCEH. Para ello se sugiere recurrir a estrategias como entrenamientos masivos, campañas de difusión y educación sobre la importancia de la RCP, RCP usando sólo las manos, etc.

- Fomentar el uso de herramientas tecnológicas, según la realidad particular de cada país y región, para aumentar la cobertura de entrenamiento en reanimación, como los recursos de autoinstrucción online, DVD o similares. En ambientes universitarios o de mayores recursos, pueden implementarse herramientas de realidad virtual para enriquecer la experiencia educativa y adquisición de habilidades.

- Implementar, especialmente en recintos de salud y de enseñanza superior, la práctica deliberada de ciclo rápido para mejorar la adquisición de habilidades en RCP.

- Impulsar sistemas de entrenamiento rápido y frecuente posteriores a entrenamientos grupales, para mejorar la retención de los conocimientos y habilidades adquiridas en los cursos tradicionales.

- Fomentar la implementación de simulación in situ en áreas de atención clínica para fortalecer los conocimientos y habilidades de los equipos clínicos durante la RCP.

\section{Brechas en las Guías para Latinoamérica}

Análisis de las deficiencias de conocimiento y propuesta de estudios ${ }^{24}$

- Maniobras y secuencia en la vía aérea ante obstrucción por cuerpo extraño. Aún faltan estudios para evaluar eficiencia de las diferentes maniobras y pasos en la liberación de la vía aérea.

- Profundidad de las compresiones torácicas en niños. No tenemos estudios experimentales y clínicos que analicen la relación entre la profundidad de la RCP y resultados hemodinámicos, recuperación de la circulación espontánea y supervivencia.
- Frecuencia respiratoria en la RCP básica y RCP avanzada. La ventilación es esencial en la RCP pediátrica. Sin embargo, las recomendaciones de frecuencia respiratoria en la RCP básica y avanzada en niños están basadas en las recomendaciones de adultos y no existen estudios clínicos prospectivos que haya comparado diferentes frecuencias. Es necesario diseñar estudios comparativos experimentales y clínicos que comparen la efectividad de distintas frecuencias respiratorias.

- Métodos de control de la ventilación. No existen estudios que aporten evidencias de los métodos más adecuados para controlar la ventilación durante la RCP en pediatría.

- Coordinación ventilación y compresiones en RCP sin intubación. En la RCP avanzada con ventilación con bolsa se recomienda realizar la ventilación coordinada con las compresiones torácicas porque se supone que si se realiza ventilación durante las compresiones torácicas no se va a lograr una suficiente ventilación pulmonar. Se requiere estudios que apoyen estas recomendaciones.

- Momento de la intubación. La intubación endotraqueal es el mejor método de control de la vía aérea y conseguir una adecuada ventilación. Son necesarios estudios prospectivos que comparen la intubación frente a la ventilación con bolsa durante la RCP en niños.

- $\quad$ Papel de otros vasopresores en la RCP en el niño. La administración de adrenalina se asocia con una mayor frecuencia de recuperación de la circulación espontánea. Son necesarios estudios que comparen la administración de adrenalina con la de otros fármacos o con la combinación de ambos.

- Dosis de energía en la desfibrilación. La dosis recomendada de desfibrilación en la RCP pediátrica no está basada en estudios de eficacia. Son necesarios estudios experimentales y clínicos en niños que comparen distintas dosis de energía en la desfibrilación en niños.

- Fármacos antiarrítmicos en los ritmos desfibrilables. Los ritmos desfibrilables son poco frecuentes en niños y el tratamiento inicial es la desfibrilación. Son necesarios estudios multicéntricos que puedan analizar este tema y evaluar eficiencia de los diferentes medicamentos utilizados, sobre todo amiodarona y lidocaína.

- Valoración de la calidad de RCP. Es necesario desarrollar estudios y evaluar resultados sobre la supervivencia y pronóstico neurológico, extender estos estudios a la región latinoamericana y con qué parámetros valorar la eficacia de la RCP avanzada. No existen estudios en niños que indiquen cuales son los datos clínicos para valorar la eficacia de la RCP. 
- ECMO y reanimación cardiopulmonar, no hay estudios en niños que hayan comparado los resultados de la ECMO-RCP con la RCP convencional, en los países en donde se disponga de esta tecnología, la posibilidad de evaluar eficacia e impacto en la sobrevida.

- Diversos estudios han encontrado diversos factores tras la recuperación de la circulación espontánea (hipoventilación, hiperventilación, hipoxia, hiperoxia e hipotensión arterial) que pueden asociarse con el pronóstico. Son necesarios estudios amplios multicéntricos que definan claramente los objetivos hemodinámicos y respiratorios tras la recuperación de la circulación que se asocian con el pronóstico en los niños.

- Control de temperatura tras el retorno de la circulación espontánea. Son necesarios estudios que analicen cuál es el objetivo de temperatura que se asocie con mejor pronóstico.

- Monitorización neurológica (eléctrica, bioquímica y de imagen) y estudios de pronóstico tras la recuperación de la circulación espontánea. En niños no hay estudios que hayan evaluado la utilidad de la monitorización multimodal para predecir el pronóstico neurológico. Es necesario planificar estudios y registros prospectivos multicéntricos para analizar los factores asociados con el pronóstico y calidad en la sobrevida.

- Entrenamiento en RCP pediátrica. Son necesarios estudios que evalúen los distintos métodos de enseñanza-aprendizaje, la forma de realizar una evaluación periódica y posibilidad de extensión a todos los niveles de formación en RCP.

- Unificación de registros. En Latinoamérica se han realizado algunos estudios de PC en niños, pero no se han desarrollado registros prospectivos que permitan evaluar siguiendo los criterios del estilo Utstein los resultados de la parada cardiaca intrahospitalaria y extrahospitalaria y la reanimación cardiopulmonar en niños.

- La investigación en RCP pediátrica es muy complicada debido a la baja frecuencia relativa de la parada cardiaca, lo inesperado de la presentación, y las importantes limitaciones éticas para desarrollar ensayos clínicos. Por ello es esencial desarrollar estudios observacionales y experimentales en modelos animales que puedan responder a las deficiencias del conocimiento en la parada cardiaca y reanimación en niños.

- Diversos estudios, fundamentalmente en países anglosajones han mostrado la que la presencia de los padres durante la RCP puede ser beneficiosa para ellos sin alterar significativamente la actuación de los reanimadores. Sin embargo, otros estudios en otras culturas y países han mostrado reticencias tanto entre los reanimadores como entre las familias. Son necesarios estudios sobre este tema en la región latinoamericana que valoren su adaptación a las características culturales y sociales en nuestros países.

\section{Conclusiones}

Se han identificado las intervenciones más importantes en RCP pediátrica adaptándolas a la realidad latinoamericana. Se han planteado diversos temas que requieren mayor investigación para el desarrollo de estrategias de prevención e intervención efectivas a nivel regional; entre ellas, el entrenamiento permanente, el registro preciso, la formación continua y la colaboración internacional son las más importantes.

\section{Conflicto de intereses}

Los autores declaran no tener conflicto de intereses.

\section{Miembros del Comité de Reanimación Cardiopulmonar SLACIP}

\section{Bernardo Alonso}

Asistente de Clínica Pediátrica, Facultad Medicina, Universidad de la República Uruguay. Montevideo, Uruguay.

\section{Manuel Alvarado Villareal}

Pediatra Intensivista. Unidad de Cuidados Intensivos, Hospital del Niño Dr. José Renán Esquivel. Ciudad de Panamá, Panamá.

\section{Santiago Campos Miño}

Pediatra Intensivista, Departamento de Pediatría, Unidad de Cuidados Intensivos Pediátricos, Hospital Metropolitano. Latin American Center for Clinical Research. Quito, Ecuador.

\section{Marcela Cuartas}

Hospital de Alta Complejidad en Red "El Cruce". Buenos Aires, Argentina.

\section{Mariana Cyunel \\ Especialista en Terapia Intensiva Pediátrica, Unidad de Cuidados Intensivos Pediátrica, Hospital de Niños Ri- cardo Gutiérrez. Buenos Aires, Argentina.}

\section{Edgard Díaz Soto}

Maestro en Administración, Especialista en Medicina de Urgencias. Ciudad de México, México. 


\section{Raffo Escalante-Kanashiro}

Médico Pediatra Intensivista. Unidad de Cuidados Intensivos, Instituto Nacional Salud del Niño, Universidad Peruana de Ciencias Aplicadas, Universidad Nacional Federico Villarreal, Lima Perú.

\section{Jesús López-Herce Cid}

Unidad de Cuidados Intensivos Pediátrica, Hospital General Universitario Gregorio Marañón. Departamento de Salud Pública y Materno-infantil, Universidad Complutense de Madrid. Madrid, España.

\section{Jaime Pezo Morales}

Departamento de Emergencias y Áreas Críticas, Servicio de Emergencia, Instituto Nacional de Salud del Niño. Lima, Perú.

\section{Norma Raúl}

Pediatra Intensivista. Hospital de Alta Complejidad en Red "El Cruce", Universidad Nacional Arturo Jauretche Florencio Varela. Buenos Aires, Argentina.

\section{Alfonso Rivera A}

Pediatra Neonatólogo. Departamento de Pediatría, Hospital Metropolitano. Presidente, Sociedad Ecuato- riana de Cuidado Intensivo Pediátrico, Coordinador del Programa NRP. Quito, Ecuador.

\section{Christian Scheu Goncalves}

Pediatra Intensivista. Unidad de Paciente Crítico Pediátrico, Hospital Clínico UC-Christus. Profesor asistente, Escuela de Medicina, Pontificia Universidad Católica de Chile. Santiago, Chile.

\section{Patricia Vásquez $\mathrm{H}$.}

Pediatra. Departamento de Pediatría, Hospital Metropolitano. Quito, Ecuador.

\section{Rocío Yerovi Santos}

Pediatra Intensivista, Unidad de Cuidados Intensivos Pediátrica, Hospital Metropolitano. Quito, Ecuador.

\section{Adriana Yock-Corrales}

Emergencióloga Pediatra, Epidemióloga. Servicio de Emergencias, Hospital Nacional de Niños Dr. Carlos Saenz Herrera. San José, Cost Rica.

\section{Mauricio Yunge}

Intensivista pediátrico. Clínica las Condes. Santiago, Chile.

\section{Referencias}

1. Alvaro Coronado RE. Síndrome de Distrés Respiratorio Agudo Pediátrico: ¿Cómo proteger los pulmones durante la ventilación mecánica? Mexico 2018.

2. Mentzelopoulos SD, Slowther AM, Fritz Z, et al. Ethical challenges in resuscitation. Intensive Care Med. 2018;44(6):703-16

3. Munoz MG, Beyda DH. An Ethical Justification for Termination of Resuscitation Protocols for Pediatric Patients. Pediatr Emerg Care. 2017;33(7):505-15.

4. Noje C, Fishe JN, Costabile PM, et al. Interhospital Transport of Children Undergoing Cardiopulmonary Resuscitation: A Practical and Ethical Dilemma. Pediatr Crit Care Med. 2017;18(10):e477-e81.

5. Morrissey PE, Monaco AP. Donation after circulatory death: current practices, ongoing challenges, and potential improvements. Transplantation. 2014;97(3):258-64.

6. Craig-Brangan KJ, Day MP. Update: Pediatric Basic Life Support and Advanced Life Support guidelines. Nursing. 2016;46(6):50-4.

7. Topjian AA, Raymond TT, Atkins D, et al Part 4: Pediatric Basic and Advanced Life
Support 2020 American Heart Association Guidelines for Cardiopulmonary Resuscitation and Emergency Cardiovascular Care. Pediatrics. 2021;147(Suppl 1).

8. Maconochie IK, Aickin R, Hazinski MF, et al. Pediatric Life Support: 2020 International Consensus on Cardiopulmonary Resuscitation and Emergency Cardiovascular Care Science With Treatment Recommendations. Resuscitation. 2020;156:A120-A55.

9. Maconochie IK, Bingham R, Eich C, et al. European Resuscitation Council Guidelines for Resuscitation 2015: Section 6. Paediatric life support. Resuscitation. 2015;95:223-48

10. Lavonas EJ, Ohshimo S, Nation K, et al. Advanced airway interventions for paediatric cardiac arrest: A systematic review and meta-analysis. Resuscitation. 2019;138:114-28.

11. Wyllie J, Bruinenberg J, Roehr CC, et al. European Resuscitation Council Guidelines for Resuscitation 2015: Section 7. Resuscitation and support of transition of babies at birth. Resuscitation. 2015;95:249-63.

12. Aziz K, Lee HC, Escobedo MB, et al. Part 5: Neonatal Resuscitation: 2020 American Heart Association Guidelines for Cardiopulmonary Resuscitation and Emergency Cardiovascular Care. Circulation. 2020;142(16_ suppl_2):S524-S50.

13. Dempsey E, Pammi M, Ryan AC, et al. Standardised formal resuscitation training programmes for reducing mortality and morbidity in newborn infants. Cochrane Database Syst Rev. 2015(9):CD009106.

14. Truhlar A, Deakin CD, Soar J, et al. European Resuscitation Council Guidelines for Resuscitation 2015: Section 4. Cardiac arrest in special circumstances. Resuscitation. 2015;95:148-201.

15. Meaney PA, Bobrow BJ, Mancini ME, et al. Cardiopulmonary resuscitation quality: [corrected] improving cardiac resuscitation outcomes both inside and outside the hospital: a consensus statement from the American Heart Association. Circulation. 2013;128(4):41735.

16. Neumar RW, Nolan JP, Adrie C, et al. Post-cardiac arrest syndrome: epidemiology, pathophysiology, treatment, and prognostication. A consensus statement from the International Liaison Committee on Resuscitation (American Heart Association, Australian and New Zealand Council on Resuscitation, European Resuscitation Council, Heart and Stroke Foundation of Canada, InterAmerican 
Heart Foundation, Resuscitation Council of Asia, and the Resuscitation Council of Southern Africa); the American Heart Association Emergency Cardiovascular Care Committee; the Council on Cardiovascular Surgery and Anesthesia; the Council on Cardiopulmonary, Perioperative, and Critical Care; the Council on Clinical Cardiology; and the Stroke Council. Circulation. 2008;118(23):2452-83.

17. Stub D, Bernard S, Duffy SJ, et al. Post cardiac arrest syndrome: a review of therapeutic strategies. Circulation. 2011;123(13):1428-35.

18. Topjian AA, de Caen A, Wainwright MS, et al. Pediatric Post-Cardiac Arrest Care: A Scientific Statement From the American Heart Association. Circulation. 2019;140(6):e194-e233.

19. Jacobs I, Nadkarni V, Bahr J, et al. Cardiac arrest and cardiopulmonary resuscitation outcome reports: update and simplification of the Utstein templates for resuscitation registries. A statement for healthcare professionals from a task force of the international liaison committee on resuscitation (American Heart Association, European Resuscitation Council, Australian Resuscitation Council, New Zealand Resuscitation Council, Heart and Stroke Foundation of Canada, InterAmerican Heart Foundation, Resuscitation Council of Southern Africa). Resuscitation. 2004;63(3):233-49.

20. Suominen P, Olkkola KT, Voipio V, et al. Utstein style reporting of in-hospital paediatric cardiopulmonary resuscitation. Resuscitation. 2000;45(1):17-25.

21. Reis AG, Nadkarni V, Perondi MB, et al. A prospective investigation into the epidemiology of in-hospital pediatric cardiopulmonary resuscitation using the international Utstein reporting style. Pediatrics. 2002;109(2):200-9.
22. Cheng A, Nadkarni VM, Mancini MB, et al. Resuscitation Education Science: Educational Strategies to Improve Outcomes From Cardiac Arrest: A Scientific Statement From the American Heart Association. Circulation. 2018;138(6):e82-e122.

23. Greif R, Bhanji F, Bigham BL, et al. Education, Implementation, and Teams: 2020 International Consensus on Cardiopulmonary Resuscitation and Emergency Cardiovascular Care Science With Treatment Recommendations. Circulation. 2020;142(16 suppl_1):S222-S83.

24. Kleinman ME, Perkins GD, Bhanji F, et al. ILCOR Scientific Knowledge Gaps and Clinical Research Priorities for Cardiopulmonary Resuscitation and Emergency Cardiovascular Care: A Consensus Statement. Resuscitation. 2018;127:13246. 